Pacific Journal of Mathematic 


\section{A REMARK ON ANALYTICITY OF FUNCTION ALGEBRAS}

\section{GLICKSBERG}

1. Let $A$ be a closed separating subalgebra of $C(X), X$ compact, with maximal ideal space $\mathfrak{M}_{A}$ and Šilov boundary $\partial_{A}$. Naturally $A$ can also be viewed as a closed subalgebra of $C\left(\mathfrak{M R}_{A}\right)$ or $C\left(\partial_{A}\right)$.

Call $A$ analytic on $X$ if the vanishing of $f \in A$ on a non-void open subset of $X$ implies $f \equiv 0$, or simply analytic if this holds for $X=$ $\mathfrak{M}_{A}$. Recently Kenneth Hoffman asked if the analyticity of $A$ on $\partial_{\boldsymbol{A}}$ implied analyticity on $\mathfrak{M}_{A}$; the present note is devoted to a counterexample. ${ }^{1}$ Evidently such an example, analytic on its Šilov boundary, must be an integral domain, so our algebra is a non-analytic integral domain.

The example was suggested by, and utilizes, an interpolation theorem of Rudin and Carleson [5,9], recently generalized by Bishop [3], which in fact permits the construction of a variety of unfamiliar tractable subalgebras of familiar algebras; consequently we shall discuss the construction in more generality than is absolutely necessary. Finally we give a slightly more complicated example which is also dirichlet.

Notation. $M(X)$ will denote the space of (finite complex regular Borel) measures $\mu$ on $X$; for such a $\mu, \mu$ is orthogonal to $A(\mu \perp A)$ if $\mu(f)=\int f d \mu=0, f$ in $A$. And $\mu_{r^{\prime}}$ will denote the usual restriction of $\mu$ to $F \subset X$, while $f \mid F$ will be the restriction of a function $f, A \mid F$ the set $\{f \mid F: f \in A\}$. An algebra $A$ will always be assumed to contain the constants.

2. Our construction is based on the following fact.

(2.1) Suppose $F$ is a closed subset of $X$, and $\mu_{F}=0$ for all $\mu$ in $M(X)$ orthogonal to $A$. Then ${ }^{2}$

$$
A \mid F=C(F)[3]
$$

(2.1.2) if $X$ is metric, $F$ is a peak set of $A$, i.e., there is an $f$ in

Received January 7, 1963. Supported in part by the National Science Foundation through Grant G22052 and in part by the Air Force Office of Scientific Research.

1 After this note was completed, I found that analyticity of $A$ on $\mathfrak{M}_{A}$ implies analyticity on $\partial_{A}$; this will appear in a subsequent paper.

2 (2.11) is Bishop's generalization of the Rudin-Carleson result mentioned before, which applies to the special case in which $A$ is the "disc algebra" and $F$ a subset of measure zero of the unit circle. (2.12) will actually be avoided in the specific examples we construct. 
$A$ with $f(F)=1$ and $|f|<1$ on $X \backslash F[7,4.8]$.

Now suppose we are given two uniformly closed algebras $A_{1}, A_{2}$, as subalgebras of $C\left(\mathfrak{M}_{1}\right), C\left(\mathfrak{M}_{2}\right)$, where $\mathfrak{M}_{i}=\mathfrak{M}_{\boldsymbol{A}_{i}}$ is metric, $i=1,2$. Further suppose $\partial_{2}=\partial_{A_{2}}$ is homeomorphic to a (compact) subset $F$ of $\partial_{1}$ satisfying the hypothesis of (2.1) with $A=A_{1}, X=\partial_{1}$, so that $A_{1} \mid F=$ $C(F)$. Identifying $F$ and $\partial_{2}$ (via some homeomorphism) we may form a compact metric space $\mathfrak{M}=\mathfrak{M}_{1} \cup \mathfrak{M}_{2}$ containing each $\mathfrak{M}_{i}$ as a subspace, with $\mathfrak{M}_{1} \cap \mathfrak{M}_{2}=F=\partial_{2}$. Now form the closed subalgebra $A$ of $C(\mathfrak{M})$ consisting of those $f$ with $f \mid \mathfrak{M}_{i}$ in $A_{i}, i=1,2$. (Since $\partial_{2} \subset \partial_{1}$, $A$ may also be viewed as a closed subalgebra of $A_{1}$.)

The consequences of (2.1) for $A$ are the following facts.

$$
\mathfrak{M}_{A}=\mathfrak{M}
$$

$$
\begin{gathered}
\partial_{A}=\partial_{1} \\
k \mathfrak{M}_{2}=\left\{f \in A: f\left(\mathfrak{M}_{2}\right)=0\right\} \text { separates the points of } \mathfrak{M} \mid \mathfrak{M}_{2} .
\end{gathered}
$$

In particular (2.4) implies there are many functions in $A$ vanishing on the (possibly void) open subset $\mathfrak{M}\left|\mathfrak{M}_{1}=\mathfrak{M}_{2}\right| \partial_{2}$ of $\mathfrak{M}=\mathfrak{M}_{A}$.

Note that since $A_{1} \mid F=C(F)$, for any $f$ in $A_{2}, f\left|\partial_{2}=f\right| F$ has an extension to $\mathfrak{M}_{1}$ in $A_{1}$; consequently $f$ itself has an extension to 'Mi in $A$. Thus

$$
A \mid \mathfrak{M}_{2}=A_{2},
$$

and $A$ separates the points of $\mathfrak{M}_{2}$. On the other hand trivially (2.6) $f$ in $A_{1}$ and $f(F)=f\left(\partial_{2}\right)=0$ imply $f$ has an extension

$$
\text { ( } \equiv 0 \text { on } \mathfrak{M R}_{2} \text { ) in } A \text {. }
$$

Now the $f$ in $A_{1}$ satisfying the hypothesis of (2.6) form an ideal $k F$ of $A_{1}$, and of course the quotient algebra $A_{1} / k F$ has the hull of $k F$ as its maximal ideal space. But $A_{1} / k F$ is naturally isomorphic to $A_{1} \mid F=C(F)$, so that $F$ is the maximal ideal space, hence the hull of .$k F$. So (as is well known and easily proved) the Banach algebra $k F$ has

$$
\partial_{k F}=\partial_{1} \backslash F=\partial_{1} \backslash \partial_{2}, \quad \mathfrak{M}_{k F}=\mathfrak{M}_{1} \backslash F .
$$

Hence from the trivial relation (2.6), $k \mathfrak{M}_{2}=\left\{f \in A: f\left(\mathfrak{M}_{2}\right)=0\right\}$ separates the points of $\mathfrak{M}_{1} \backslash F=\mathfrak{M} \mid \mathfrak{M}_{2}$, yielding (2.4), and separates any element of $\mathfrak{M} \backslash \mathfrak{M}_{2}$ from one of $\mathfrak{M}_{2}$. Since $A$ separates the points of $\mathfrak{M}_{2}$ by (2.5), $A$ separates $\mathfrak{M}$, and $\mathfrak{M}$ is a subspace of $\mathfrak{M}_{\boldsymbol{A}}$. Moreover by (2.6) $k F$ and $k \mathfrak{M}_{2}$ are isomorphic, whence $\partial_{k \mathfrak{M}}=\partial_{1} \mid \partial_{2}$, so that

$$
\partial_{1} \mid \partial_{2} \subset \partial_{A}
$$

The remainder of (2.2) now follows by a standard argument: if a multiplicative linear functional $\varphi$ on $A$ vanishes on $k \mathfrak{M}_{2}$, hence corresponds to an element of $\mathfrak{M R}_{A / k \mathfrak{M}_{2}}$, then the isomorphism of $A / k \mathfrak{M}_{2}$ and $A \mid \mathfrak{M}_{2}=A_{2}$ shows $\varphi$ arises from a point in $\mathfrak{M}_{2} \subset \mathfrak{M}$. But if $\varphi$ does not vanish on $k \mathrm{MR}_{2}$ it provides a nonzero functional on this algebra, 
hence on $k F$, and (since $\mathfrak{M}_{k F}=\mathfrak{M}_{1} \backslash F$ ) we have some $x$ in $\mathfrak{M}_{1}$ for which $\varphi(f)=f(x), f$ in $k \mathfrak{M k}_{2}$. Choosing $f$ in $k \mathfrak{M}_{2}$ with $f(x)=\varphi(f)=1$, we have $f g$ in $k \mathfrak{M l}_{2}$ for any $g$ in $A$, so $\varphi(g)=\varphi(f g)=f g(x)=g(x)$.

For (2.3), we already have $\partial_{A} \subset \partial_{1}$ (since $f \in A$ assumes its maximum modulus on $\partial_{1}$ by the definition of $A$ ) and $\partial_{1} \mid \partial_{2} \subset \partial_{A}$ by (2.8). Consequently (2.3) follows immediately if $F=\partial_{2}$ is nowhere dense in $\partial_{1}$ (as in the case of our examples to follow) since $\partial_{1}=\left(\partial_{1} \mid \partial_{2}\right)^{-} \subset \partial_{A}$.

For the general case we need only show $x$ in $\partial_{2}$ lies in $\partial_{A}$, and for this part of the argument we shall restrict our attention to $\partial_{1}$ and regard $A$ and $A_{1}$ as subalgebras of $C\left(\partial_{1}\right), A_{2}$ as one of $C\left(\partial_{2}\right)$. By (2.12) (with $X=\partial_{1}, F=\partial_{2}$ and $A_{1}$ our algebra) we have an element $f$ of $A_{1}$ peaking on $F$, so $f(F)=1,|f|<1$ on $\partial_{1} \mid F$; and of course $f \in A$. For our $x$ in $\partial_{2}$ and any open neighborhood $U$ of $x$ in $\partial_{1}$ we know there is a $g_{2}$ in $A_{2}$ assuming its maximum modulus over $\partial_{2}-1$ say-only within $\partial_{2} \cap U$, and by (2.5) $g_{2}$ has an extension $g$ in $A$. Moreover for some $\varepsilon>0,\left|g_{2}\right|<1-\varepsilon$ on $\partial_{2} \mid U$, so $|g|<1-\varepsilon$ on some open subset $V$ of $\partial_{1}$ containing $\partial_{2} \mid U$. Since $\partial_{2}$ is contained in the open subset $U \cup V$ of $\partial_{1}$, sup $\left|f\left(\partial_{1} \mid(U \cup V)\right)\right|<1$, so $\left|f^{n} g\right|<1-\varepsilon$ on $\partial_{1} \mid(U \cup V)$ for some $n$, while $\left|f^{n} g\right| \leqq|g|<1-\varepsilon$ on $V$. Thus $\left|f^{n} g\right|<1-\varepsilon$ on $\partial_{1} \mid U$; since $f^{n} g=g$ on $\partial_{2}$ the element $f^{n} g$ of $A$ assumes its maximum modulus 1 only within $U$, whence $x \in \partial_{A}$ and $\partial_{2} \subset \partial_{A}$ as desired.

2.2 RemaRK. (2.2)-(2.4) apply to a more general construction; for with $F \subset \partial_{1}$ having $\mu_{F}=0$ for all $\mu$ in $M\left(\partial_{1}\right)$ orthogonal to $A_{1}$ as before, and $\rho$ any (not one-to-one) continuous map of $F$ onto $\partial_{2}$ we can set

$$
A=\left\{f \in A_{1}: f \mid F \in A_{2} \circ \rho\right\}
$$

and again arrive at the same conclusions. Here, of course, in forming $\mathfrak{M}$ there is some identification of points in $F$, while $\partial_{A}$ is $\partial_{1}$ with just such identifications. (An appropriate modification of (4.1) below can also be obtained in this setting.)

3. We can now write down our example. Let $A_{1}$ be the disc algebra of all functions continuous in the disc $D=\{z:|z| \leqq 1\}$ and analytic on $|z|<1$. Let $A_{2}$ be Rudin's algebra [10] of all functions continuous on the Riemann sphere $S$ and analytic off a compact perfect 0-dimensional subset $E$ of the plane with $E \cap U$ void or of positive plane measure for each open $U$. $\operatorname{Then}^{3} E=\partial_{2}$ and $\mathfrak{M}_{2}=S$ [2].

3 This follows from the argument of $[\mathbf{1 0}$, p. 826]. For if $U$ is open in $S$ and $E \cap U \neq \phi$ is open and closed in $E$ then-with $E \cap U$ in place of $E-[\mathbf{1 0}]$ shows there are non-constant $f$ in $C(S)$ analytic off $E \cap U$, hence elements of $A$ assuming their maximum modulus only within $E \cap U$. 
Now pick a Cantor set $F$ of measure 0 on the unit circle $T^{1}=\partial_{1}$ so $\mu_{F}=0$ for each $\mu$ in $M\left(T^{1}\right)$ orthogonal to $A_{1}$ by the F. and M. Riesz theorem [8]. $E=\partial_{2}$ and $F$ are homeomorphic so we may identify these sets as before, in effect tacking $S$ onto $D$ along $F$. Our algebra $A$ on the resulting space $\mathfrak{M}=D \cup S$ consists of all functions continuous on an open subset of $\partial_{A}=\partial_{1}=T^{1}$ must vanish on $\mathfrak{M}$ and analytic off $T^{1}$.

Now $S \backslash E=\mathfrak{M}_{2} \backslash F$ is a non-void open subset of $\mathfrak{M}_{A}=\mathfrak{M}$ on which nonzero elements of $A$ do vanish by (2.4); but an $f$ in $A$ which vanishes on all of $T^{1}$, being analytic on the interior of $D$, whence $f \equiv 0$.

4. We conclude with a modification of our example in which our nonanalytic integral domain is also a dirichlet algebra on its Silov boundary [8]. In order to see the example is dirichlet, we require the following additional information, which holds in the context of $\S 2$.

Let $A, A_{1}, A_{2}$ again be as in $\S 2$. Let $A_{i}^{\perp}$ denote the measures on $\partial_{i}$ orthogonal to $A_{i}$, and $A^{\perp}$ those on $\partial_{A}=\partial_{1}$ orthogonal to $A$. (Since $\partial_{2} \subset \partial_{1}$, we shall view $A_{2}^{\perp}$ as consisting of measures on $\partial_{1}$.) Then

$$
A^{\perp}=A_{1}^{\perp}+A_{2}^{\perp}
$$

(4.1) is a consequence of an argument of Browder and Wermer [4]. To obtain it, consider the weak* closed subspaces $A^{\perp}, A_{i}^{\perp}$ of the dual $M\left(\partial_{1}\right)$ of $C\left(\partial_{1}\right)$. Clearly $A_{i}^{\perp} \subset A^{\perp}$, so $A_{1}^{\perp}+A_{2}^{\perp} \subset A^{\perp}$. On the other hand any $f$ in $C\left(\partial_{1}\right)$ orthogonal to $A_{1}^{\perp}+A_{2}^{\perp}$ has $f \mid \partial_{i}$ in $A_{i} \mid \partial_{i}$, so $f \mid \partial_{i}$ has an extension $g_{i}$ in $A_{i}, i=1,2$; and evidently $g_{1}$ and $g_{2}$ combine to yield an extension $g$ of $f, g \in A$. So $f \in A \mid \partial_{1}$, which shows $A_{1}^{\perp}+A_{2}^{\perp}$ is weak* dense in $A^{\perp}$.

So it suffices to prove $A_{1}^{\perp}+A_{2}^{\perp}$ is weak* closed in $M\left(\partial_{1}\right)$. But by hypothesis $\mu_{\partial_{2}}=0$ for all $\mu$ in $A_{1}^{\perp}$, so $\mu$ in $A_{1}^{\perp}$ and $\nu$ in $A_{2}^{\perp}$ are mutually singular, and $\|\mu+\nu\|=\|\mu\|+\|\nu\|$. Consequently the argument of Browder and Wermer [4] applies to complete the proof of (4.1).

Now let $Z^{2}$ be the lattice points in the plane, $\alpha$ an irrational real number, and $H$ the half-space of $Z^{2}$ of all $(m, n)$ with

$$
m \alpha+n \geqq 0 \text {. }
$$

Let $A_{1}$ be the closed algebra of continuous functions on the torus $T^{2}$ spanned by the characters of $T^{2}$ corresponding to the elements of the semigroup $H$; alternatively $A_{1}$ consists of those $f$ in $C\left(T^{2}\right)$ with Fourier coefficients vanishing off $H$. A description of $\mathfrak{M}_{1}$ can be found in [1]; but here we only need the fact that $\partial_{1}=T^{2}[1]$, and that $A_{1}$ is a dirichlet algebra on $T^{2}$.

Let $F$ be the subset $T^{1} \times\{1\}$ of $T^{2}$. Then from an extension of the F. and M. Riesz theorem obtained recently by $K$. de Leeuw and the 
author [6] we have ${ }^{4}$ (i) $\mu_{F}=0$ for all $\mu$ in $M\left(T^{2}\right)$ orthogonal to $A_{1}$ [6, Th. 3.1], while (ii) any $f$ in $A_{1}$ which vanishes on an open subset of $T^{2}$ vanishes identically [6, Th. 4.1]. From (i) we can apply our construction, identifying $F$ with the boundary of the disc $D$, taking $A_{2}$ as the disc algebra. The resulting algebra $A$ again contains nonzero elements vanishing on an open subset of $\mathfrak{M}_{A}$-the interior of $D$ - and again is analytic on $\partial_{A}=T^{2}$ by (ii).

And $A$ is dirichlet on $T^{2}$ by (4.1): for if $\lambda$ is any real measure in $M\left(T^{2}\right)$ orthogonal to $A$, so that $\lambda=\mu_{1}+\mu_{2}, \mu_{i}$ in $A_{i}^{\perp}$, then $\mu_{2}=\lambda_{F}$, $\mu_{1}=\lambda_{F^{\prime}}$, by (i). Consequently $\mu_{i}$ is a real measure on $\partial_{i}$ orthogonal to $A_{i}$, hence zero since $A_{i}$ is dirichlet on $\partial_{i}$.

Finally, note that $A$ has a simple description as a subalgebra of $C\left(T^{2}\right)$ : viewing $T^{1}$ as the reals $\bmod 2 \pi, A$ consists of all $f$ with

$$
\begin{array}{lr}
\int_{0}^{2 \pi} \int_{0}^{2 \pi s} f(\theta, \varphi) e^{-i(m \theta+n \varphi)} d \theta d \varphi=0, & m \alpha+n<0, \\
\int_{0}^{2 \pi} f(0, \varphi) e^{-i n \varphi} d \varphi=0, & n<0 .
\end{array}
$$

\section{REFERENCES}

1. R. Arens and I. M. Singer, Generalized analytic functions, Trans. Amer. Math. Soc., 81 (1956), 379-393.

2. R. Arens, The maximal ideals of certain function algebras, Pacific J. Math., 8 (1958), 641-648.

3. E. Bishop, A general Rudin-Carleson theorem, Proc. Amer. Math. Soc., 13 (1962), 13 (1962), 140-143.

4. A. Browder and J. Wermer, Some algebras of functions on an arc, J. Math. Mech., 12 (1963), 119-130.

5. L. Carleson, Representations of continuous functions, Math. Z. 66 (1957), 447-451.

6. K. de Leeuw and I. Glicksberg, Analytic measures on compact groups, Quasi-invariance and analyticity of measures on compact groups, Acta Math., 109 (1963), 179-205.

7. I. Glicksberg, Measures orthogonal to algebras and sets of antisymmetry, Trans. Amer. Math. Soc., 105 (1962), 415-435.

8. K. Hoffman, Banach Spaces of Analytic Functions, Englewood Cliffs, N.J., 1962.

9. W. Rudin, Boundary values of continuous analytic functions, Proc. Amer. Math. Soc., 7 (1956), 808-811.

10. - Subalgebras of spaces of continuous functions, Proc. Amer. Math. Soc., 7 (1956), 825-830.

UNIVERSITY OF WASHINGTON

${ }^{4}$ Here the map $\psi$ of [6] taking $Z^{2}$ into $R$ is $(m, n) \rightarrow m \alpha+n$. 



\section{PACIFIC JOURNAL OF MATHEMATICS}

\section{EDITORS}

RalPh S. Phillips

Stanford University

Stanford, California

M. G. Arsove

University of Washington

Seattle 5 , Washington
J. Dugundu

University of Southern California

Los Angeles 7, California

Lowell J. Paige

University of California

Los Angeles 24, California

\section{ASSOCIATE EDITORS}
E. F. BECKENBACH
D. DERRY
H. L. ROYDEN
E. G. STRAUS
T. M. CHERRY
M. OHTSUKA
E. SPANIER
F. WOLF

\section{SUPPORTING INSTITUTIONS}

\author{
UNIVERSITY OF BRITISH COLUMBIA \\ CALIFORNIA INSTITUTE OF TECHNOLOGY \\ UNIVERSITY OF CALIFORNIA \\ MONTANA STATE UNIVERSITY \\ UNIVERSITY OF NEVADA \\ NEW MEXICO STATE UNIVERSITY \\ OREGON STATE UNIVERSITY \\ UNIVERSITY OF OREGON \\ OSAKA UNIVERSITY \\ UNIVERSITY OF SOUTHERN CALIFORNIA
}

\author{
STANFORD UNIVERSITY \\ UNIVERSITY OF TOKYO \\ UNIVERSITY OF UTAH \\ WASHINGTON STATE UNIVERSITY \\ UNIVERSITY OF WASHINGTON \\ AMERICAN MATHEMATICAL SOCIETY \\ CALIFORNIA RESEARCH CORPORATION \\ SPACE TECHNOLOGY LABORATORIES \\ NAVAL ORDNANCE TEST STATION
}

Mathematical papers intended for publication in the Pacific Journal of Mathematrcs should be typewritten (double spaced), and the author should keep a complete copy. Manuscripts may be sent to any one of the four editors. All other communications to the editors should be addressed to the managing editor, L. J. Paige at the University of California, Los Angeles 24, California.

50 reprints per author of each article are furnished free of charge; additional copses may be obtained at cost in multiples of 50 .

The Pacific Journal of Mathematics is published quarterly, in March, June, September, and December. Effective with Volume 13 the price per volume (4 numbers) is $\$ 18.00$; single issues, $\$ 5.00$. Special price for current issues to individual faculty members of supporting institutions and to individual members of the American Mathematical Society: $\$ 8.00$ per volume; single issues $\$ 2.50$. Back numbers are available.

Subscriptions, orders for back numbers, and changes of address should be sent to Pacific Journal of Mathematics, 103 Highland Boulevard, Berkeley 8, California.

Printed at Kokusai Bunken Insatsusha (International Academic Printing Co., Ltd.), No. 6 , 2-chome, Fujimi-cho, Chiyoda-ku, Tokyo, Japan.

\section{PUBLISHED BY PACIFIC JOURNAL OF MATHEMATICS, A NON-PROFIT CORPORATION}

The Supporting Institutions listed above contribute to the cost of publication of this Journal, but they are not owners or publishers and have no responsibility for its content or policies. 


\section{Pacific Journal of Mathematics}

\section{Vol. 13, No. $4 \quad$ June, 1963}

Dallas O. Banks, Bounds for eigenvalues and generalized convexity ........... 1031

Jerrold William Bebernes, A subfunction approach to a boundary value problem for

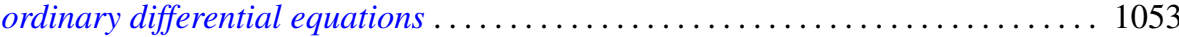

Woodrow Wilson Bledsoe and A. P. Morse, A topological measure construction . . . 1067

George Clements, Entropies of several sets of real valued functions . . . . . . . . . 1085

Sandra Barkdull Cleveland, Homomorphisms of non-commutative *-algebras . . . . . 1097

William John Andrew Culmer and William Ashton Harris, Convergent solutions of

ordinary linear homogeneous difference equations . . . . . . . . . . . . . . . 1111

Ralph DeMarr, Common fixed points for commuting contraction mappings . . . . . . 1139

James Robert Dorroh, Integral equations in normed abelian groups . . . . . . . . 1143

Adriano Mario Garsia, Entropy and singularity of infinite convolutions . . . . . . . 1159

J. J. Gergen, Francis G. Dressel and Wilbur Hallan Purcell, Jr., Convergence of extended Bernstein polynomials in the complex plane ................. 1171

Irving Leonard Glicksberg, A remark on analyticity of function algebras . . . . . . 1181

Charles John August Halberg, Jr., Semigroups of matrices defining linked operators

with different spectra ................................. 1187

Philip Hartman and Nelson Onuchic, On the asymptotic integration of ordinary

differential equations . . . . . . . . . . . . . . . . . . . . . . . . . . . . 1193

Isidore Heller, On a class of equivalent systems of linear inequalities . . . . . . . . . 1209

Joseph Hersch, The method of interior parallels applied to polygonal or multiply

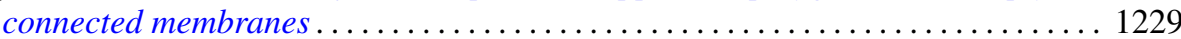

Hans F. Weinberger, An effectless cutting of a vibrating membrane . . . . . . . . . . 1239

Melvin F. Janowitz, Quantifiers and orthomodular lattices ....

Samuel Karlin and Albert Boris J. Novikoff, Generalized convex inequalities . .

Tilla Weinstein, Another conformal structure on immersed surfaces of negative

curvature.

Gregers Louis Krabbe, Spectral permanence of scalar operators

Shige Toshi Kuroda, Finite-dimensional perturbation and a representaion of

scattering operator.

Marvin David Marcus and Afton Herbert Cayford, Equality in certain

inequalities

Joseph Martin, A note on uncountably many disks .

Eugene Kay McLachlan, Extremal elements of the convex cone of semi-norms . . . . 1335

John W. Moon, An extension of Landau's theorem on tournaments . .

Louis Joel Mordell, On the integer solutions of $y(y+1)=x(x$

Kenneth Roy Mount, Some remarks on Fitting's invariants .....

Miroslav Novotný, Über Abbildungen von Mengen ............

Robert Dean Ryan, Conjugate functions in Orlicz spaces.

John Vincent Ryff, On the representation of doubly stochastic operators . . . . . . . . 1379

Donald Ray Sherbert, Banach algebras of Lipschitz functions .

James McLean Sloss, Reflection of biharmonic functions across analytic boundary

conditions with examples.

L. Bruce Treybig, Concerning homogeneity in totally ordered, connected topological space....

John Wermer, The space of real parts of a function algebra...

James Juei-Chin Yeh, Orthogonal developments of functionals and related theorems

in the Wiener space of functions of two variables......... 